\title{
VARIABILIDADE FENOTÍPICA EM GENÓTIPOS DE ACEROLA
}

\author{
JOSÉ EVERALDO GOMES ${ }^{2}$, DILERMANDO PERECIN³, \\ ANTONIO BALDO GERALDO MARTINS ${ }^{4}$ e EDUARDO JOSÉ DE ALMEIDA ${ }^{5}$
}

\begin{abstract}
RESUMO - As medidas de variabilidade fenotípica, correlações, efeitos diretos e indiretos na aceroleira (Malpighia emarginata DC.) da região de Itápolis, SP, objetivaram direcionar o processo seletivo de genótipos. Os parâmetros comprimento e largura média de folhas, altura de planta, diâmetro de copa, peso médio de fruto, peso médio de polpa/fruto, peso médio de 20 frutos e peso médio de polpa por 20 frutos mostraram diferenças quanto a genótipos e épocas. Em acidez, tamanho e largura média de fruto, as diferenças são exclusivas em relação a épocas, sugerindo ações de condições climáticas. A altura é um bom parâmetro tipificador na diferenciação e seleção de genótipos. As condições meteorológicas podem influenciar marcadamente em caracteres tecnológicos (acidez) e em medidas dos frutos. Destacaram-se as associações positivas entre comprimento de folhas com tamanho e peso de frutos e as correlações negativas destes com vitamina C; seguida de correlações positivas e significativas de tamanho com largura de frutos. Dos efeitos diretos sobre vitamina $C$, relacionam-se os efeitos de Brix e diâmetro de copa como positivos. Contudo, nas condições estudadas, as medidas das folhas estão associadas positivamente com tamanho e peso dos frutos e negativamente com vitamina C. Além disso, observa-se que as medidas dos frutos associam-se positivamente com os caracteres de peso e rendimento, que estão negativamente associados com vitamina C. A seleção sobre Brix pode direcionar ganhos em polpa e em vitamina $\mathrm{C}$.
\end{abstract}

Termos para indexação: Malpighia emarginata, métodos de melhoramento, seleção, critérios de seleção, ganho genético, condições meteorológicas, melhoramento de plantas.

\section{PHENOTYPIC VARIABILITY IN WEST INDIAN CHERRY GENOTYPES}

\begin{abstract}
The measures in the phenotypic variability, correlation, direct and indirect effects in the West Indian cherry (Malpighia emarginata DC.), from Itápolis, São Paulo State, Brazil, aimed to subsidize the management of the selective process in the genotypes. The parameters length and width of leaves, height of plant, diameter of canopy, weight medium of fruit, weight of pulp/fruit, weight medium of 20 fruits and weight medium of pulp of 20 fruits, showed differences for genotypes and date of evaluation. The differences of acidity, size and width medium of fruit are exclusive for dates, suggesting actions of climatic conditions. The height is a good parameter leading to the differentiation for selection of genotypes. The meteorological conditions can influence remarkably the acidity and dimensions of the fruit. Positive associations among leaf length and the parameters that define size and weight of fruits and negative correlation of these parameters with vitamin $C$ were observed; so as positive correlation of size and width of fruits. Brix and canopy diameter were positively correlated with vitamin C. Measures of the leaves are associated positively with size and weight of the fruits and negatively with vitamin $\mathrm{C}$ in the studied conditions. The measures of the fruits are directly associated with the characters of weight and yield, that are inversely associated with vitamin $\mathrm{C}$. The selection on Brix can manage the gain in pulp and vitamin $\mathrm{C}$.
\end{abstract}

Index terms: Malpighia emarginata, breeding methods, selection, selection criteria, genetic gain, meteorological elements, plant breeding.

\footnotetext{
${ }^{1}$ Aceito para publicação em 19 de janeiro de 2000.

Extraído da tese de doutorado apresentada pelo primeiro autor à Universidade Estadual Paulista (UNESP). Projeto financiado pela FAPESP.

${ }^{2}$ Biól., M.Sc., Dep. de Ciências Exatas, FCAV, UNESP, Rod. Paulo Donato Castellani, km 5, CEP 14870-000 Jaboticabal, SP.
}

\author{
${ }^{3}$ Eng. Agrôn., Prof. Titular, Dep. de Ciências Exatas, FCAV, \\ UNESP. Bolsista do CNPq. E-mail: perecin@fcav.unesp.br \\ ${ }^{4}$ Eng. Agrôn., Dr., Prof. Assistente, Dep. de Horticultura, FCAV, \\ UNESP. E-mail: baldo@fcav.unesp.br \\ ${ }^{5}$ Eng. Agrôn., Dep. de Horticultura, FCAV, UNESP.
}




\section{INTRODUÇÃO}

A acerola (Malpighia emarginata DC.), conhecida como cereja das Antilhas, é uma planta arbustiva com dois a três metros de altura, de ramos densos e espalhados, folhas opostas e flores em cachos axilares com três a cinco unidades (Couceiro, 1985). Foi considerada, por Araújo \& Minami (1994), a planta da década de 90 , por causa da riqueza de seus frutos em vitamina $\mathrm{C}$.

O mercado consumidor da acerola no Brasil é predominante nas regiões quentes, em virtude do hábito de ingestão de sucos. A demanda ocorre no mercado interno sob as formas de frutos in natura ou congelados como polpa. O mercado externo, que busca na acerola principalmente a fonte de vitamina $\mathrm{C}$, consome mais o produto na forma de polpa.

Referindo-se ao binômio vitamina C e luminosidade, Murphy (1938) refere-se aos autores Bracewell et al. (1931), Birch et al. (1933), McHenry \& Graham (1935) e Todhunter (1936), que indicam a luz como um dos muitos fatores que influenciam a produção de vitamina C. Winston (1947), Brown (1955) e Malavolta et al. (1956) observaram que a quantidade dessa vitamina aumenta em frutos de espécies vegetais expostos diretamente ao sol e a altas intensidades luminosas. Também Nakasone et al. (1966), analisando o efeito da radiação solar no conteúdo de ácido ascórbico nos frutos da aceroleira, em cinco níveis de luminosidade, concluíram que a redução no teor desse ácido em plantas com $75 \%$ de sombreamento foi de $17 \%$, quando comparadas com as que não sofreram restrição da luz solar.

Arostequi \& Pennock (1955) obtiveram um conteúdo médio em vitamina $\mathrm{C}$ de $2 \%$ e um rendimento de $59 \%$ a $73 \%$, em relação ao peso do fruto. Arostequi et al. (1955) destacaram que o teor de vitamina $C$ no fruto varia em razão da época da colheita.

Outra conotação na variação da vitamina $\mathrm{C}$ em frutos de acerola foi observada por Teixeira \& Azevedo (1995) quando relataram que uma vez satisfeita as exigências térmicas, maior disponibilidade hídrica proporciona maior produção de ácido ascórbico pela planta até um certo limite, a partir do qual o excesso hídrico é prejudicial; chuvas excessivas provocam a formação de frutos aquosos e menos ricos em açúcar e vitamina C (Simão, 1971).
A variabilidade existente nos pomares brasileiros é acentuada, notavelmente em regiões onde o plantio estabeleceu-se através de pés francos. Assim, objetivou-se neste trabalho avaliar a variabilidade fenotípica em genótipos de acerola e analisar as correlações, efeitos diretos e indiretos entre as variáveis estudadas, para que se processe e direcione a seleção dos genótipos que atendam aos mercados de consumo interno e externo.

\section{MATERIAL E MÉTODOS}

O experimento foi conduzido em um pomar comercial não-irrigado, de aproximadamente seis anos, na região de Itápolis, São Paulo, em área caracterizada como Argissolo Vermelho-Amarelo e inserida nas seguintes coordenadas: $21^{\circ} 35^{\prime}$ de latitude Sul e $48^{\circ} 47^{\prime}$ de longitude Oeste e $560 \mathrm{~m}$ de altitude (Dicionário..., 1970).

O pomar era composto por um total de 3.000 plantas com grande variabilidade fenotípica. Dessas, as 12 melhores foram selecionadas para estudo, sendo devidamente marcadas, podadas e manejadas convenientemente.

Realizaram-se avaliações mensais em folhas, frutos e plantas, no período de dezembro/97 a março/98. Em folhas, foram medidos comprimento e largura. Em frutos, foram avaliados, tamanho, largura, peso e peso de polpa. Também foram avaliados peso de 20 frutos, peso de polpa de 20 frutos, peso médio de 20 frutos, em três amostras; peso médio de polpa em três amostras de 20 frutos, rendimento de polpa em três amostras, quantidade de vitamina $\mathrm{C}$ (em $100 \mathrm{~mL}$ de suco pelo método do iodo), acidez e Brix. Na planta, foram medidos altura, diâmetro de copa, crescimento total em 20 ramos e crescimento médio de ramos.

As análises de variância foram obtidas por meio do PROC GLM do SAS; modelo com genótipos e épocas, sem interações. Para avaliação das correlações lineares entre os parâmetros estudados utilizou-se o PROC CORR do SAS, segundo o método de Pearson (Steel \& Torrie, 1960). Quanto aos coeficientes de caminho ou trilha, foram obtidos através do PROC REG do SAS, com variáveis padronizadas ((variável - média)/desvio-padrão), segundo o método de $\mathrm{Li}$ (1978).

\section{RESULTADOS E DISCUSSÃO}

Na Tabela 1, as análises de variância mostram diferenças significativas em genótipos e em épocas, nos parâmetros comprimento e largura de folhas, altura de planta, diâmetro de copa, peso médio de fruto, peso médio de polpa/fruto, peso médio de 20 fru- 


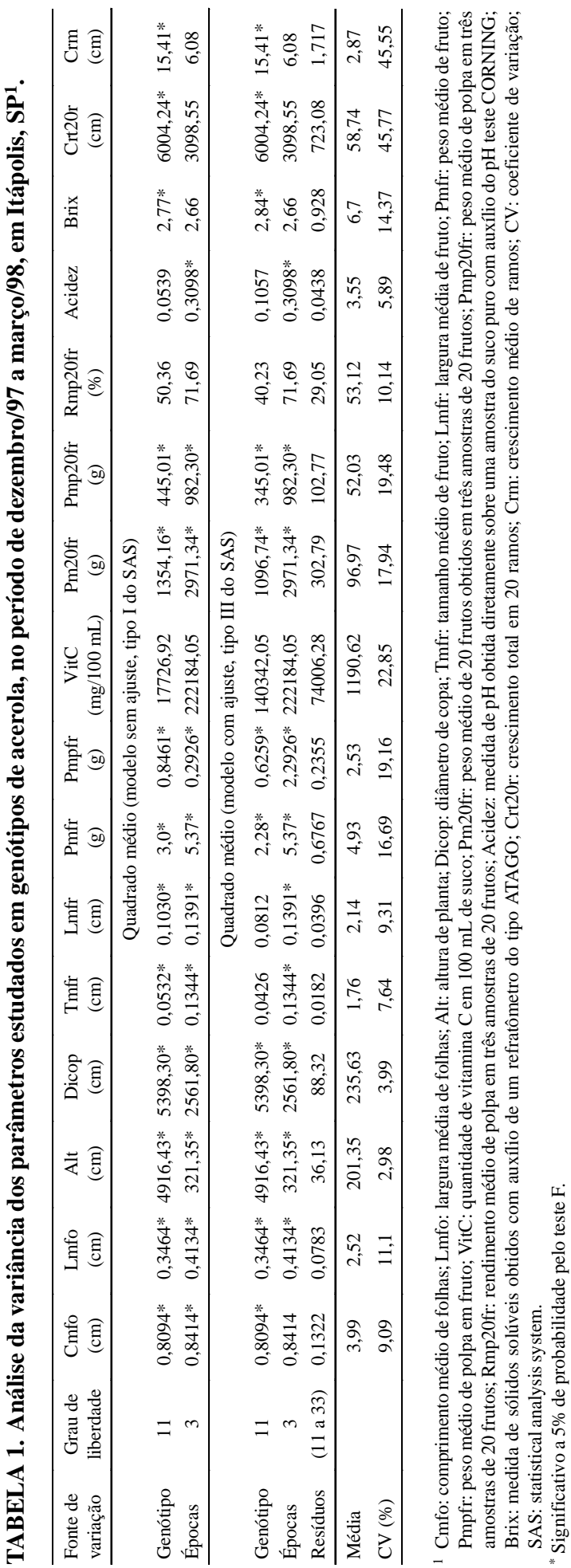

tos e peso médio de polpa de 20 frutos, sugerindo variação dos caracteres entre genótipos e dentro de genótipos nas épocas.

Diferenças significativas em Brix, crescimento total de 20 ramos e crescimento médio de ramos, somente em genótipos, sugerem que tais caracteres são menos sensíveis a variações nas condições meteorológicas no período avaliado.

Em acidez, tamanho e largura de frutos, foram verificadas diferenças significativas em épocas, sugerindo que caracteres tecnológicos (acidez) e medidas do fruto, podem ser influenciados por fatores climáticos, no período estudado. A vitamina $\mathrm{C}$ varia em razão da luminosidade (Winston, 1947; Brown, 1955; Malavolta et al., 1956) e da disponibilidade hídrica, até certo limite (Teixeira \& Azevedo, 1995).

Os rendimentos de $47,8 \%$ (genótipo 5) a $58,9 \%$ (genótipo 6) estão abaixo da faixa de rendimentos encontrados por Arostequi \& Pennock (1955), de $59 \%$ a $73 \%$, atribuindo-se à menor variabilidade na população selecionada ou à falta de irrigação (Tabela 2).

Em altura de planta, verificam-se diferenças significativas em genótipos e épocas (Tabela 1) e grande variabilidade entre genótipos (Tabela 2), sugerindo que seja parâmetro adequado para diferenciação e seleção, desde que se considere a facilidade na colheita, quantidade de vitamina $\mathrm{C}$, rendimento em polpa e número de safras/ano.

Em peso médio de fruto, o genótipo 8 mostrou-se superior, podendo ser indicado para frutos de mesa e venda in natura. Todavia, no período estudado, registrou-se menor número de safras, favorecendo a indicação do genótipo 5 como potencialmente adequado, uma vez que também possui frutos grandes e boas características.

$\mathrm{Na}$ Tabela 3, são considerados os valores individuais de correlações entre os parâmetros nas épocas em que foram amostrados e os valores com médias ajustadas, obtidas com o LSMEANS do SAS, ou seja, a média dos valores das quatro épocas (Tabela 4). Destacam-se as associações positivas de comprimento de folhas com os parâmetros que definem o tamanho e o peso do fruto, pela sua importância no processo seletivo indireto.

Em tamanho médio de frutos verificam-se correlações positivas significativas quanto a largura de 
TABELA 2. Médias de parâmetros avaliados em 12 genótipos de acerola no período de dezembro/97 a março/98, em Itápolis, SP1 .

\begin{tabular}{|c|c|c|c|c|c|c|c|c|c|c|c|c|c|c|c|c|}
\hline Genótipo & $\begin{array}{l}\text { Cmfo } \\
(\mathrm{cm})\end{array}$ & $\begin{array}{l}\text { Lmfo } \\
(\mathrm{cm})\end{array}$ & $\begin{array}{c}\text { Alt } \\
(\mathrm{cm})\end{array}$ & $\begin{array}{c}\text { Dicop } \\
(\mathrm{cm})\end{array}$ & $\begin{array}{l}\text { Tmfr } \\
(\mathrm{cm})\end{array}$ & $\begin{array}{l}\mathrm{Lmfr} \\
(\mathrm{cm})\end{array}$ & $\begin{array}{c}\text { Pmfr } \\
(\mathrm{g})\end{array}$ & $\begin{array}{l}\text { Pmpfr } \\
(\mathrm{g})\end{array}$ & $\begin{array}{c}\mathrm{VitC} \\
(\mathrm{mg} / 100 \mathrm{~mL})\end{array}$ & $\begin{array}{c}\text { Pm20fr } \\
(\mathrm{g})\end{array}$ & $\begin{array}{l}\text { Pmp20fr } \\
\text { (g) }\end{array}$ & $\begin{array}{c}\text { Rmp20fr } \\
(\%)\end{array}$ & Acidez & Brix & $\begin{array}{c}\text { Crt20r } \\
(\mathrm{cm})\end{array}$ & $\begin{array}{l}\mathrm{Crm} \\
(\mathrm{cm})\end{array}$ \\
\hline 1 & $3,54 \mathrm{de}$ & $2,47 \mathrm{abcd}$ & $203,8 \mathrm{de}$ & $232,5 b c$ & $1,58 \mathrm{a}$ & $1,95 \mathrm{a}$ & $3,36 a b$ & $1,27 \mathrm{ab}$ & $943,3 \mathrm{a}$ & $58,5 \mathrm{ab}$ & $28,5 b$ & $49,1 \mathrm{a}$ & $3,42 \mathrm{a}$ & $5,25 \mathrm{~b}$ & $42,8 b c$ & $2,14 b c$ \\
\hline 2 & $4,48 \mathrm{abc}$ & 2,79abc & $192,5 \mathrm{ef}$ & $235 \mathrm{bc}$ & $1,86 \mathrm{a}$ & $2,13 \mathrm{a}$ & $5,26 a b$ & $2,74 a b$ & $873,5 \mathrm{a}$ & $96,2 \mathrm{ab}$ & $54,9 \mathrm{ab}$ & $57,7 \mathrm{a}$ & $3,82 \mathrm{a}$ & $6,25 \mathrm{ab}$ & $36,5 \mathrm{c}$ & $1,06 \mathrm{c}$ \\
\hline 3 & 3,88abcde & 2,31abcd & $131,3 \mathrm{~h}$ & $155 \mathrm{e}$ & $1,71 \mathrm{a}$ & $2,03 a$ & $4,20 \mathrm{ab}$ & $2,37 a b$ & $1285,0 \mathrm{a}$ & $81,7 \mathrm{ab}$ & $48,1 \mathrm{ab}$ & $58,5 \mathrm{a}$ & $3,30 \mathrm{a}$ & $6,75 \mathrm{ab}$ & $19,0 \mathrm{c}$ & $0,95 \mathrm{c}$ \\
\hline 5 & 4,13abcde & 2,73abcd & $277,5 \mathrm{a}$ & $250 \mathrm{bc}$ & $1,82 \mathrm{a}$ & $2,42 \mathrm{a}$ & $5,59 \mathrm{ab}$ & $2,37 a b$ & $1306,2 \mathrm{a}$ & $122,0 \mathrm{a}$ & $57,2 \mathrm{ab}$ & $47,8 \mathrm{a}$ & $3,24 \mathrm{a}$ & $8,32 \mathrm{a}$ & $145,3 \mathrm{ab}$ & 7,30ab \\
\hline 6 & 4,15abcde & 2,67abcd & $212,5 \mathrm{bcd}$ & $250 \mathrm{bc}$ & $1,70 \mathrm{a}$ & $2,17 \mathrm{a}$ & $4,71 \mathrm{ab}$ & $2,66 a b$ & $1167,4 \mathrm{a}$ & $88,8 \mathrm{ab}$ & $52,9 \mathrm{ab}$ & $58,9 \mathrm{a}$ & $3,21 \mathrm{a}$ & $6,70 \mathrm{a}$ & $103,0 a b c$ & $5,20 \mathrm{abc}$ \\
\hline 7 & 4,35abcd & 2,42abcd & $225 b$ & $278,8 \mathrm{a}$ & $1,87 \mathrm{a}$ & $2,27 \mathrm{a}$ & $5,86 a c$ & $2,94 \mathrm{a}$ & $1206,4 \mathrm{a}$ & $108,6 \mathrm{ab}$ & $59,9 \mathrm{ab}$ & $55,0 \mathrm{a}$ & $3,22 \mathrm{a}$ & $6,90 \mathrm{ab}$ & $10,3 \mathrm{c}$ & $0,52 \mathrm{c}$ \\
\hline 8 & $4,55 \mathrm{ab}$ & $2,89 \mathrm{ab}$ & 206,3 cde & $228,8 \mathrm{c}$ & $1,80 \mathrm{a}$ & $2,43 a$ & $6,96 a$ & $3,55 \mathrm{a}$ & $853,5 \mathrm{a}$ & $138,3 \mathrm{a}$ & $81,6 a$ & $58,8 \mathrm{a}$ & $3,07 \mathrm{a}$ & $7,28 \mathrm{ab}$ & $9,8 \mathrm{c}$ & $0,49 \mathrm{c}$ \\
\hline 10 & $3,34 \mathrm{e}$ & $2,19 \mathrm{~cd}$ & $181,3 \mathrm{f}$ & $245 b c$ & $1,48 \mathrm{a}$ & $2,0 \mathrm{a}$ & $3,79 b c$ & $2,02 \mathrm{ab}$ & $1163,5 \mathrm{a}$ & $74,2 \mathrm{ab}$ & $40,8 \mathrm{~b}$ & $54,8 \mathrm{a}$ & $3,12 \mathrm{a}$ & $5,73 \mathrm{ab}$ & $10,8 \mathrm{c}$ & $0,54 \mathrm{c}$ \\
\hline 11 & $3,76 \mathrm{bcde}$ & 2,5abcd & $203,8 \mathrm{de}$ & $235 b c$ & $1,84 \mathrm{a}$ & $2,07 \mathrm{a}$ & $4,63 a b$ & $2,35 \mathrm{ab}$ & $1001,5 \mathrm{a}$ & $95,3 \mathrm{ab}$ & $56,0 \mathrm{ab}$ & $58,4 \mathrm{a}$ & $3,21 \mathrm{a}$ & $5,85 \mathrm{ab}$ & $40,5 b c$ & $2,03 b c$ \\
\hline 12 & $4,67 \mathrm{a}$ & $2,99 \mathrm{a}$ & $166,3 \mathrm{~g}$ & $178,8 \mathrm{~d}$ & $1,60 \mathrm{a}$ & $1,84 \mathrm{a}$ & $3,26 \mathrm{~b}$ & $1,64 \mathrm{~b}$ & $1343,0 \mathrm{a}$ & $67,9 b$ & $35,4 \mathrm{~b}$ & $49,5 \mathrm{a}$ & $3,20 \mathrm{a}$ & $6,23 \mathrm{ab}$ & $114,9 \mathrm{abc}$ & $5,8 \mathrm{abc}$ \\
\hline
\end{tabular}

1 As médias da mesma coluna, seguidas das mesmas letras, não diferem entre si pelo teste de Tukey a $5 \%$ de probabilidade; Cmfo: comprimento médio de folhas; Lmfo: laroura média de folhas; Alt: altura de planta; Dicop: diâmetro de copa; Tmfr: tamanho médio de fruto; Lmfr: largura média de fruto; Pmfr: peso médio de fruto; Pmpfr: peso médio de polpa em fruto; VitC: quantidade de vitamina C em 100 mL de suco; Pm20fr: peso médio de 20 frutos obtidos em três amostras de 20 frutos; Pmp20fr: peso médio de polpa em três amostras de 20 frutos; Rmp 20 fr: rendimento médio de polpa em três amostras de 20 frutos; Acidez: medida de $\mathrm{pH}$ obtida diretamente sobre uma amostra do suco puro com auxílio do $\mathrm{pH}$ teste CORNING; Brix: medida de sólidos solúveis obtidos com auxílio de um refratômetro do tipo ATAGO; Crt20r: crescimento total em 20 ramos; Crm: crescimento médio de ramos.

TABELA 3. Correlações entre os parâmetros estudados nos genótipos de acerola entre todos os valores coletados, sem ajuste para épocas, no período de dezembro/97 a março/98, em Itápolis, $\mathrm{SP}^{\mathbf{1}}$.

\begin{tabular}{|c|c|c|c|c|c|c|c|c|c|c|c|c|c|c|c|}
\hline Parâmetro & Lmfo & Alt & Dicop & Tmfr & Lmfr & Pmfr & Pmpfr & Acidez & Brix & VitC & Pm20fr & Pmp20fr & Rmp20fr & Crt20r & $\mathrm{Crm}$ \\
\hline Cmfo $(\mathrm{cm})$ & $0,89 *$ & 0 & $-0,32 *$ & $0,37 *$ & 0,27 & $0,37^{*}$ & 0,29 & $0,36^{*}$ & 0,30 & $-0,22$ & 0,34 & 0,29 & $-0,08$ & 0 & $-0,01$ \\
\hline Lmfo $(\mathrm{cm})$ & & 0,06 & $-0,34 *$ & 0,28 & 0,32 & 0,28 & 0,13 & 0,29 & 0,26 & $-0,21$ & 0,26 & 0,17 & $-0,24$ & 0,01 & 0 \\
\hline Alt $(\mathrm{cm})$ & & & 0,72 & 0,30 & $0,53 *$ & $0,43^{*}$ & 0,22 & $-0,02$ & 0,34 & 0,02 & $0,49 *$ & 0,34 & $-0,24$ & 0,29 & 0,29 \\
\hline Dicop $(\mathrm{cm})$ & & & & 0,16 & 0,32 & 0,31 & 0,22 & $-0,09$ & 0,19 & 0,04 & 0,30 & 0,26 & 0,02 & $-0,02$ & $-0,03$ \\
\hline $\operatorname{Tmfr}(\mathrm{cm})$ & & & & & $0,83^{*}$ & $0,89^{*}$ & $0,81^{*}$ & 0,25 & $-0,18$ & $-0,55^{*}$ & $0,83^{*}$ & $0,84 *$ & 0,36 & $-0,04$ & $-0,04$ \\
\hline $\operatorname{Lmfr}(\mathrm{cm})$ & & & & & & $0,90^{*}$ & $0,77^{*}$ & 0,06 & 0,06 & $-0,36$ & $0,88^{*}$ & $0,81^{*}$ & 0,13 & 0 & 0,02 \\
\hline $\operatorname{Pmfr}(\mathrm{g})$ & & & & & & & $0,93^{*}$ & 0,17 & $-0,02$ & $-0,45^{*}$ & $0,95^{*}$ & $0,93^{*}$ & 0,29 & $-0,09$ & $-0,09$ \\
\hline Pmpfr (g) & & & & & & & & 0,16 & $-0,17$ & $-0,46^{*}$ & $0,88^{*}$ & $0,93 *$ & $0,45^{*}$ & $-0,07$ & $-0,09$ \\
\hline Acidez & & & & & & & & & $-0,01$ & $-0,27$ & 0,16 & 0,16 & 0,05 & $-0,11$ & $-0,16$ \\
\hline Brix & & & & & & & & & & 0,60 & 0,06 & $-0,07$ & $-0,35$ & $-0,04$ & 0 \\
\hline $\operatorname{VitC}(\mathrm{mg} / 100 \mathrm{~mL})$ & & & & & & & & & & & $-0,38^{*}$ & $-0,45^{*}$ & $-0,39 *$ & 0,16 & 0,19 \\
\hline $\operatorname{Pm} 20 \mathrm{fr}(\mathrm{g})$ & & & & & & & & & & & & $0,93^{*}$ & 0,2 & $-0,04$ & $-0,04$ \\
\hline Pmp20fr (g) & & & & & & & & & & & & & $0,52 *$ & $-0,08$ & $-0,09$ \\
\hline Rmp20fr (\%) & & & & & & & & & & & & & & $-0,24$ & $-0,27$ \\
\hline Crt20r (cm) & & & & & & & & & & & & & & & $0,99^{*}$ \\
\hline
\end{tabular}

${ }^{1}$ Cmfo: comprimento médio de folhas; Lmfo: largura média de folhas; Alt: altura de planta; Dicop: diâmetro de copa; Tmfr: tamanho médio de fruto; Lmfr: largura média de fruto; Pmfr: peso médio de fruto; Pmpfr: peso médio de polpa em fruto; VitC: quantidade de vitamina $\mathrm{C}$ em $100 \mathrm{~mL}$ de suco; Pm20fr: peso médio de 20 frutos obtidos em três amostras de 20 frutos; Pmp20fr: peso médio de polpa em três amostras de 20 frutos; Rmp20fr: rendimento médio de polpa em três amostras de 20 frutos; Acidez: medida de pH obtida diretamente sobre uma amostra do suco puro com auxílio do pH teste CORNING; Brix: medida de sólidos solúveis obtidos com auxílio de um refratômetro do tipo ATAGO; Crt20r: crescimento total em 20 ramos; Crm: crescimento médio de ramos.

* Significativo a $5 \%$ de probabilidade. 


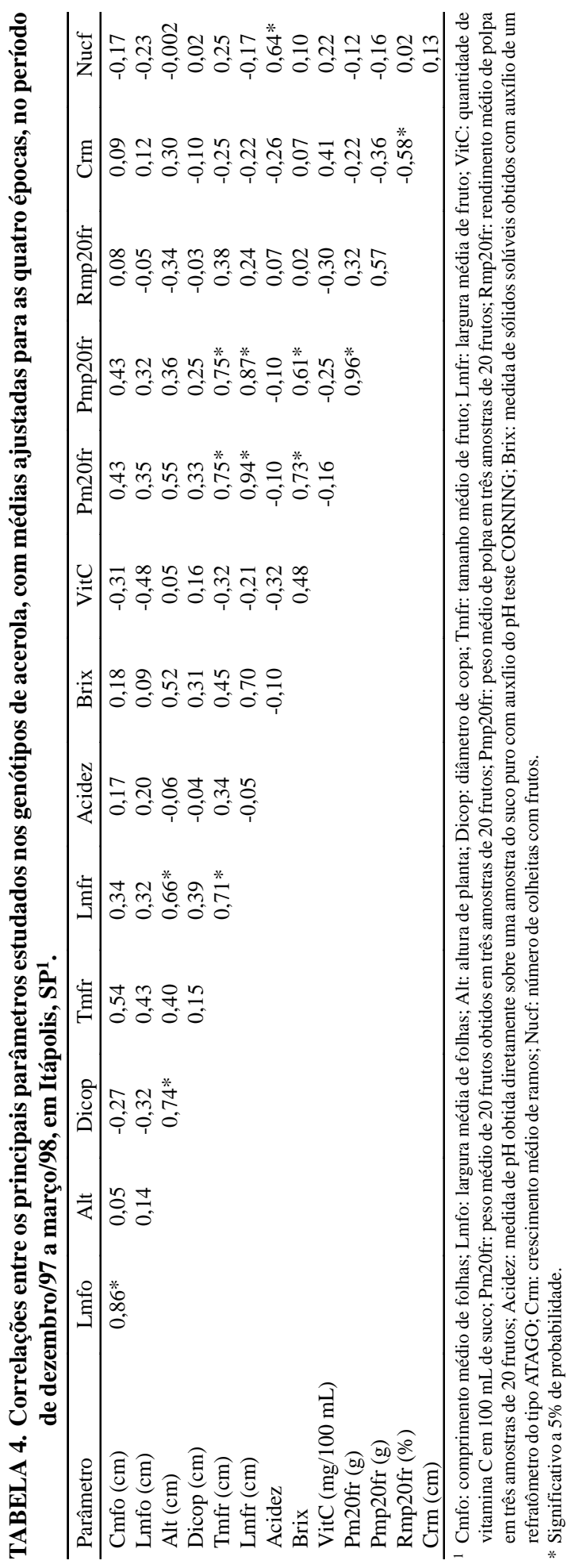

folha, peso médio de fruto, peso médio de polpa/fruto, peso médio de 20 frutos e com peso médio de polpa de 20 frutos, e negativas e significativas com vitamina $\mathrm{C}$; importantes no direcionamento da seleção, pois ao se levar em conta somente tamanho e peso de fruto, poderá reduzir a vitamina $\mathrm{C}$.

Em peso médio de fruto constatam-se correlações positivas e significativas do caráter com peso médio de polpa/fruto e peso médio de polpa em 20 frutos, e negativas e significativas com vitamina $\mathrm{C}$. Em peso médio de polpa/fruto ocorrem correlações significativas e positivas com peso médio de 20 frutos, peso médio de polpa em 20 frutos, rendimento médio de polpa em 20 frutos, e negativas e significativas com vitamina $C$; demonstrando que os caracteres estudados em frutos estão, na maioria, inversamente associados com a vitamina $\mathrm{C}$.

Nos caracteres tecnológicos em frutos, como Brix, destacam-se as correlações positivas e significativas com vitamina $\mathrm{C}$, e negativa não-significativa com rendimento médio de polpa em 20 frutos; mostrando que Brix e vitamina C estão inversamente associados com o rendimento de polpa nos frutos, e que Brix pode ajudar no processo seletivo de genótipo com alto teor de vitamina C. Isso é possível, desde que considere na escolha do genótipo tamanho e largura de frutos, para assegurar a produção em polpa, balanceando os efeitos favoráveis e desfavoráveis, pois a vitamina $\mathrm{C}$ apresenta correlações significativas negativas com peso médio de 20 frutos, peso médio de polpa em 20 frutos e rendimento médio de polpa em 20 frutos.

Quanto ao Brix, associa-se com vitamina C, numa certa magnitude, porém sem significância, e correlaciona-se de modo positivo significativo com peso médio de 20 frutos $(r=0,73)$, peso médio de polpa em 20 frutos $(r=0,61)$, deixando nessa conotação a possibilidade de selecionar em Brix para atingir maiores ganhos em polpa e em vitamina $\mathrm{C}$ (Tabela 4).

Para o direcionamento do processo seletivo, estimaram-se os coeficientes de caminho entre os principais parâmetros, desdobrando-os em efeitos diretos e indiretos via componentes que apresentaram significância com o efeito direto (Tabelas 5 e 6).

$\mathrm{Na}$ comparação entre o fator causal Brix, que mostrou um efeito direto de 0,96 , com a vitamina $C$, e correlação (Brix e vitamina $C, r=0,60$ ), destacam- 
TABELA 5. Correlações entre os parâmetros estudados e a quantidade de vitamina C; estimativas dos efeitos diretos dos caracteres e efeitos indiretos de Brix e diâmetro de copa (Dicop) sobre vitamina $C$ via seus componentes, em genótipos de acerola mensurados no período de dezembro/97 a março/98, em Itápolis, $\mathbf{S P}^{1}$.

\begin{tabular}{|c|c|c|c|c|}
\hline \multirow[t]{2}{*}{ Parâmetro } & \multirow{2}{*}{$\begin{array}{l}\text { Correlação } \\
\text { com } \\
\text { vitamina C }\end{array}$} & \multirow{2}{*}{$\begin{array}{l}\text { Efeito direto } \\
\text { na } \\
\text { vitamina } \mathrm{C}\end{array}$} & \multicolumn{2}{|c|}{ Efeito indireto } \\
\hline & & & $\begin{array}{c}\text { Via Brix } \\
\text { na vitamina } C\end{array}$ & $\begin{array}{c}\text { Via Dicop } \\
\text { na vitamina C }\end{array}$ \\
\hline Brix & $0,60 *$ & $0,96^{*}$ & $\mathrm{NA}^{2}$ & 0,1824 \\
\hline Pe20 & $-0,45$ & $-0,56$ & 0,0112 & $-0,1680$ \\
\hline Rmp20fr & $-0,39$ & 0,16 & $-0,0560$ & 0,0032 \\
\hline Cmfo & $-0,22$ & 0,23 & 0,0690 & $-0,0736$ \\
\hline Lmfo & $-0,21$ & 0,04 & 0,0104 & $-0,0136$ \\
\hline Alt & 0,02 & $-0,98$ & $-0,3332$ & $-0,7056$ \\
\hline Dicop & 0,04 & $0,99 *$ & 0,1881 & NA \\
\hline Tmfr & $-0,55$ & 0,09 & $-0,0162$ & 0,0144 \\
\hline Lmfr & $-0,36$ & 0,14 & 0,0084 & 0,0448 \\
\hline Acidez & $-0,27$ & $-0,31$ & 0,0031 & 0,0279 \\
\hline Crt20r & 0,16 & 0,51 & $-0,0204$ & $-0,0102$ \\
\hline
\end{tabular}

${ }^{1}$ Brix: medida de sólidos solúveis obtido com auxílio de um refratômetro do tipo ATAGO; Pe20: peso de 20 frutos; Rmp20fr: rendimento médio de polpa em três amostras de 20 frutos; Cmfo: comprimento médio de folhas; Lmfo: largura média de folhas; Alt: altura de planta; Dicop: diâmetro de copa; Tmfr: tamanho médio de fruto; Lmfr: largura média de fruto; Acidez: medida de $\mathrm{pH}$ obtida diretamente sobre uma amostra do suco puro com auxílio do pH teste CORNING; Crt20r: crescimento total em 20 ramos.

2 Não aplicável.

* Significativo a $5 \%$ de probabilidade pelo teste $\mathrm{F}$.

TABELA 6. Correlações entre os parâmetros estudados e peso médio de polpa em 20 frutos (Pmp20fr); estimativas dos efeitos diretos dos caracteres e efeitos indiretos de rendimento médio de polpa em 20 frutos (Rmp20fr) sobre Pmp20fr via seus componentes em genótipos de acerola, no período de dezembro/97 a março/98, em Itápolis, $\mathbf{S P}^{\mathbf{1}}$.

\begin{tabular}{lccc}
\hline Parâmetro & $\begin{array}{c}\text { Correlação } \\
\text { com } \\
\text { Pmp20fr }\end{array}$ & $\begin{array}{c}\text { Efeito direto } \\
\text { no } \\
\text { Pmp20fr }\end{array}$ & $\begin{array}{c}\text { Efeito indireto } \\
\text { via Rmp20fr } \\
\text { no Pmp20fr }\end{array}$ \\
\hline Brix & $-0,07$ & 0,130 & $-0,0455$ \\
Pe20 & $0,93^{*}$ & 0,780 & 0,2340 \\
Rmp20fr & $0,52^{*}$ & $0,420^{*}$ & $\mathrm{NA}^{2}$ \\
Cmfo & 0,29 & $-0,180$ & 0,0144 \\
Lmfo & 0,17 & 0,230 & $-0,0552$ \\
Alt & 0,34 & 0,010 & $-0,0024$ \\
Dicop & 0,26 & 0,020 & 0,0004 \\
Tmfr & $0,84^{*}$ & $-0,040$ & $-0,0144$ \\
Lmfr & $0,81^{*}$ & 0,003 & 0,0004 \\
Acidez & 0,16 & 0,010 & 0,0005 \\
Crt20r & $-0,08$ & 0,100 & $-0,0240$ \\
\hline
\end{tabular}

${ }^{1}$ Brix: medida de sólidos solúveis obtido com auxílio de um refratômetro do tipo ATAGO; Pe20: peso de 20 frutos; Rmp20fr: rendimento médio de polpa em três amostras de 20 frutos; Cmfo: comprimento médio de folhas; Lmfo: largura média de folhas; Alt: altura de planta; Dicop: diâmetro de copa; Tmfr: tamanho médio de fruto; Lmfr: largura média de fruto; Acidez: medida de $\mathrm{pH}$ obtida diretamente sobre uma amostra do suco puro com auxílio do $\mathrm{pH}$ teste CORNING; Crt20r: crescimento total em 20 ramos.

2 Não aplicável.

* Significativo a 5\% de probabilidade pelo teste F. se os valores positivos em ambos, indicando que Brix é importante no processo seletivo de genótipos promissores (Tabela 5).

Para diâmetro de copa, o efeito direto de 0,99, com vitamina $\mathrm{C}$, é marcante, porém a correlação é baixa (Tabela 5 ).

Para atender à demanda de consumo da polpa de acerola e obter boa cotação da polpa no mercado internacional, é importante associar a esta teor em vitamina $\mathrm{C}$, e para o produtor um genótipo que seja produtivo, além do teor em vitamina C. Dessa maneira, foram obtidas estimativas dos coeficientes de caminho também para peso médio de polpa em 20 frutos (Tabela 6).

Inicialmente, observam-se as significâncias nas correlações positivas de peso médio de polpa em 20 frutos com peso de 20 frutos, rendimento médio de polpa em 20 frutos, tamanho médio de fruto e comprimento médio de fruto. Quando comparadas com os efeitos diretos, destaca-se o rendimento médio de polpa em 20 frutos pela proximidade entre os valores, explicando a correlação e indicando que a seleção direta sobre rendimento é efetivo para ganho em polpa. De maneira similar, destaca-se o efeito direto de peso de 20 frutos, porém não-significativo, mas de alta magnitude, indicando que são relacionados, por isso efetivos no processo de seleção. 


\section{CONCLUSÕES}

1. As diferenças em peso médio de fruto, peso médio de polpa/fruto, peso médio de 20 frutos, peso médio de polpa em 20 frutos, acidez, tamanho e largura de frutos são resultantes de ações com fatores climáticos e de genótipos.

2. Altura de planta é adequado para diferenciação e seleção.

3. As condições meteorológicas têm ações marcantes em caracteres tecnológicos (acidez) e em medidas dos frutos.

4. Os caracteres comprimento e largura de folhas estão associados positivamente com os parâmetros que definem o tamanho e o peso do fruto.

5. O direcionamento do processo seletivo em tamanho e peso do fruto implica redução da vitamina $C$.

6. Os caracteres tamanho e largura de frutos estão associados positivamente com peso e rendimento de polpa.

7. A seleção sobre Brix direciona o ganho em polpa e vitamina $\mathrm{C}$.

8. Dos genótipos analisados, o 5 reúne maior número de parâmetros favoráveis.

\section{AGRADECIMENTO}

À FAPESP, pela concessão da bolsa e apoio financeiro ao projeto de doutorado.

\section{REFERÊNCIAS}

ARAÚJO, P.S.R. de; MINAMI, K. Acerola. Campinas : Fundação Cargill, 1994. 81p.

AROSTEQUI, F.; ASENJO, C.F.; MUINIZ, A.J.; ALEMANY, L. Observations and data on a promising selection of the West Indian cherry Malpighia punicifolia L. University of Puerto Rico Journal of Agriculture, Rio Piedras, v.39, n.2, p.51-56, 1955.
AROSTEQUI, F.; PENNOCK, W. La acerola. Rio Piedras : Universidad de Puerto Rico-Estación Experimental Agrícola, 1955. 9p. (Publicación Miscelánea, 15).

BROWN, G.B. The ascorbic acid content of tomatoes as related to illumination. American Society for Horticultural Science. Proceedings, Alexandria, v.68, n.65, p.342-347, 1955.

COUCEIRO, E.M. Curso de extensão sobre a cultura da acerola. Recife : UFRPE, 1985. 45p.

DICIONÁRIO geográfico brasileiro. Porto Alegre : Globo, 1970. p.236.

LI, C.C. First course in population genetics. Pacific Grove : Boxwood, 1978. 631p.

MALAVOLTA, E.J.; GURGELL, J.T.A.; SOBRO, J.S. Ascorbic acid content in fruit of Myrciaria glomerata Bergs. Nature, London, v.178, n.4530, p.424, 1956.

MURPHY, E. Vitamin C and light. American Society for Horticulture Science. Proceedings, Alexandria, v.36, p.498-499, 1938.

NAKASONE, H.Y.; MIYASHITA, R.K.; YAMANE, G.M. Factors affecting ascorbic acid content of the acerola (Malpighia glabra L.). American Society for Horticulture Science. Proceedings, Alexandria, v.49, p.161-166, 1966.

SIMÃO, S. Manual de fruticultura. São Paulo : Agronômica Ceres, 1971. p.477-485.

STEEL, R.G.D.; TORRIE, J.H. Principles and procedures of statistics. New York : McGraw-Hill, 1960. 481p.

TEIXEIRA, A.H. de C.; AZEVEDO, P.V. Índice do clima para o cultivo da acerola. Pesquisa Agropecuária Brasileira, Brasília, v.30, n.12, p.1403-1410, dez. 1995.

WINSTON, J.R. Vitamin C content and juice quality of exposed and shaded citrus fruit. American Society for Horticulture Science. Proceedings, Alexandria, v.52, n.60, p.63-67, 1947. 